\title{
Analyzing the Frontier scientific issue applying Classical Separation Variable Method
}

\author{
Yuee $\mathrm{Li}^{1,}$ a , Zhonglei $\mathrm{Mei}^{1}$, Aning $\mathrm{Ma}^{1}$, Zhong Wang ${ }^{1}$, Lin Cheng ${ }^{1}$ \\ ${ }^{1}$ School of Information Science and Engineering, Lanzhou University, Lanzhou, 730000, China \\ aemail: liyuee@Izu.edu.cn
}

Keywords: Research-oriented teaching; Mathematics Physics Method; Plasmonic waveguide; Separation Variable Method

\begin{abstract}
In this paper, we introduce surface plasmons, a hot issue in the Frontiers of Science and Technology into the teaching process of "Mathematical Physics Method" course. We analyzed extensively metal nanowires, a typical plasmonic waveguide combining the separation method and numerical solution method. Dispersion Equation of guided modes by Metal Nanowires and Electromagnetic Field are investigated. On this basis, the single-mode transmission conditions of metal nanowires and the mode size of the fundamental mode are analyzed. Our practice has proved that this kind of teaching methods integrating the frontier of science and technology is of great significance to improve students' learning interest, arouse students' initiative and creativity in classroom learning, and cultivate their ability of scientific research and innovation.
\end{abstract}

\section{Introduction}

"Mathematical Physics Method" is an important professional basic course for electronic information and communication engineering discipline. Different from the "Advanced Mathematics" course, as a bridge of mathematics applied to physics, it will provide the basic mathematical treatment for the follow-up courses of "Electromagnetic Field Theory", "Microwave Technology", "Signal and System" and "Optical Fiber Communication". This course is composed of two parts: complex variable function and mathematical physics equations. The separation variable method in three commonly used coordinate is significant for solving various mathematical equations, especially in the latter two coordinate systems involved in special functions. Because of the initial contact with complex special function concept, it is very difficult for students to understand these basic concepts [1].

We have been working to improve teaching method for this sort of course which covers a lot of mathematical and physical knowledge. A series of reform ideas are explored such as introducing the latest scientific research trends to students for solving specific scientific problems applying complex special function, combining with numerical simulation tools to enable students to master the concept in the process of using new knowledge and stirring up their enthusiasm and creativity of this course by visual pictures and animations [2, 3].

Many students have such understanding that the knowledge of mathematical physics is not new and out of line with the current science and technology development. Therefore, in the ongoing teaching reform, we have continually tried to introduce the hot issues of scientific research field into the classroom and service teaching [4]. In this paper, we use the classical mathematical physics method to solve metal nanowire, one type of Surface Plasmon Waveguide (SPW). SPW has become a hot issue in recent years due to its strong local field enhancement characteristics, breaking through the diffraction limit. The transmission and processing of optical information at the nanometer scale can be achieved and it has great application potential in various fields of optics [5, 6]. In addition, outstanding electric field confinement of SPW makes it widely used in the high sensitive biochemical detection, sensing and new type of light sources. Here, surface modes of metal nanowires are analyzed by using the separation variable method in cylindrical coordinate system. At the same time, the electric field graphs of several modes are plotted with numerical simulation software COMSOL. The students can profoundly understand the concept and significance of 
surface modes while they learn the variable separation method in cylindrical coordinate system. Meanwhile, we can cultivate student's awareness of scientific research and innovation and also promote the teaching reform of mathematical physics course.

\section{The Structure and Dispersion Equation of Metal Nanowires}

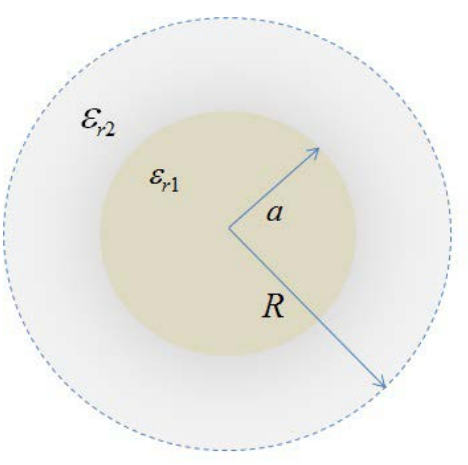

Fig. 1 Theoretical model of the metal nanowire $\left(\operatorname{Re}\left(\varepsilon_{r 1}\right)<0, \varepsilon_{r 2}>0\right)$

Metal nanowire could be regarded as an infinite long and straight metal nanowire with relative permittivity $\varepsilon_{r 1}<0$ embraced by rolling dielectric coaxial cylinders with relative permittivity $\varepsilon_{r 2}>0$, internal radius $\mathrm{R}_{\mathrm{in}}=a$ and external radius $\mathrm{R}_{\text {out }}=\infty$. Because the electric field of surface modes are confined on the interface of the metal and dielectric, this approximation $R_{\text {out }}=\infty$ is reasonable . The theoretical model of the metal nanowire is illustrated in Figure 1.

Considering optical modes propagating along $\mathrm{z}$ in SPPs waveguides, the longitudinal components in metal nanowires can be written in the forms

$$
\begin{aligned}
& \tilde{E}_{z}=E_{z}(x, y) e^{-j \beta z} \\
& \tilde{H}_{z}=H_{z}(x, y) e^{-j \beta z}
\end{aligned}
$$

Then, The longitudinal components in metal nanowires $E_{z 1}, H_{z 1} \quad(r<a)$ 和 $E_{z 2}, H_{z 2} \quad(r>a)$ meets equations:

$$
\begin{array}{ll}
\nabla_{T}^{2} E_{z 1}+\left(k_{1}^{2}-\beta^{2}\right) E_{z 1}=0 & (0 \leq r \leq a) \\
\nabla_{T}^{2} H_{z 1}+\left(k_{1}^{2}-\beta^{2}\right) H_{z 1}=0 & (0 \leq r \leq a) \\
\nabla_{T}^{2} E_{z 2}+\left(k_{2}^{2}-\beta^{2}\right) E_{z 2}=0 & (r \geq a) \\
\nabla_{T}^{2} H_{z 2}+\left(k_{2}^{2}-\beta^{2}\right) H_{z 2}=0 & (r \geq a)
\end{array}
$$

Where, $k_{1}=k_{0} n_{1}, k_{2}=k_{0} n_{2}, n_{1}=\sqrt{\varepsilon_{r 1}}$ and $n_{2}=\sqrt{\varepsilon_{r 2}}$ are refractive indexes of metal core and dielectric cladding respectively. For SPPs modes supported by SPPs waveguides, $\frac{\beta}{k_{0}}>n_{1}{ }^{2}, n_{2}{ }^{2}$, and namely $k_{0}{ }^{2} n_{1}^{2}-\beta^{2}<0, k_{0}{ }^{2} n_{2}^{2}-\beta^{2}<0$, Both Eq. 2 and Eq.3 are imaginary argument Bessel equations and the solutions, define

$$
\begin{gathered}
U_{1}^{2}=a^{2}\left(\beta^{2}-k_{0}{ }^{2} n_{1}^{2}\right) \\
U_{2}{ }^{2}=a^{2}\left(\beta^{2}-k_{0}{ }^{2} n_{2}{ }^{2}\right) \\
V^{2}=U_{1}{ }^{2}-U_{2}{ }^{2}=a^{2} k_{0}{ }^{2}\left(n_{2}{ }^{2}-n_{1}{ }^{2}\right)
\end{gathered}
$$

Where, $U_{1}^{2}>0, U_{2}^{2}>0$ Considering natural boundary conditions at $r=0, r \rightarrow \infty$ and continual 
boundary conditions $E_{z 1}=\left.E_{z 2}\right|_{r=a}, H_{z 1}=\left.H_{z 2}\right|_{r=a}$, the solutions are

$$
\begin{gathered}
E_{z 1}=A^{\prime} \frac{I_{m}\left(\frac{U_{1}}{a} r\right)}{I_{m}\left(U_{1}\right)} e^{j m \varphi} e^{-j \beta z} \\
H_{z 1}=B^{\prime} \frac{I_{m}\left(\frac{U_{1}}{a} r\right)}{I_{m}\left(U_{1}\right)} e^{j m \varphi} e^{-j \beta z} \\
E_{z 2}=A^{\prime} \frac{K_{m}\left(\frac{U_{2}}{a} r\right)}{K_{m}\left(U_{2}\right)} e^{j m \varphi} e^{-j \beta z} \\
H_{z 2}=B^{\prime} \frac{K_{m}\left(\frac{U_{2}}{a} r\right)}{K_{m}\left(U_{2}\right)} e^{j m \varphi} e^{-j \beta z}
\end{gathered}
$$

Using Longitudinal field methods, other components in metal and dielectric can be derived from $E_{z}$ and $H_{z}$ Applying the boundary conditions $E_{\varphi 1}=E_{\varphi 2}, H_{\varphi 1}=H_{\varphi 2}$ at $r=a$, we can obtain the dispersion equations of TM modes while $\mathrm{m}=0$

$$
\frac{n_{1}^{2} I_{1}\left(U_{1}\right)}{U_{1} I_{0}\left(U_{1}\right)}+\frac{n_{2}^{2} K_{1}\left(U_{2}\right)}{U_{2} K_{0}\left(U_{2}\right)}=0
$$

and the dispersion equations of HE modes while $m \neq 0$

$$
(\beta m)^{2}\left(\frac{1}{U_{1}^{2}}-\frac{1}{U_{2}^{2}}\right)^{2}=-k_{0}^{2}\left(\frac{n_{1}^{2}}{U_{1}} \frac{I_{m}^{\prime}\left(U_{1}\right)}{I_{m}\left(U_{1}\right)}-\frac{n_{2}^{2}}{U_{2}} \frac{K_{m}{ }^{\prime}\left(U_{2}\right)}{K_{m}\left(U_{2}\right)}\right)\left(\frac{1}{U_{2}} \frac{K_{m}{ }^{\prime}\left(U_{2}\right)}{K_{m}\left(U_{2}\right)}-\frac{1}{U_{1}} \frac{I_{m}{ }^{\prime}\left(U_{1}\right)}{I_{m}\left(U_{1}\right)}\right)
$$

Important parameters of all modes can be obtained, such as the effective indexes, the transmission distances, the electric/magnetic field distribution etc. by solving Eq. 6 and Eq. 7. The field confinement can also be discussed using mode field diameter directly. Table 1 displays effective indexes of $\mathrm{TM}_{0}$ mode and some $\mathrm{HE}_{\mathrm{m}}$ modes guided by silver nanowires (silica cladding $\varepsilon_{\mathrm{r} 2}=1.452$ )

\begin{tabular}{|c|c|c|c|c|c|c|c|}
\hline$a$ & TM & $\mathrm{HE}_{1}$ & $\mathrm{HE}_{2}$ & $\mathrm{HE}_{3}$ & $\mathrm{HE}_{4}$ & $\mathrm{HE}_{5}$ & $\begin{array}{c}\text { Number } \\
\text { of } \\
\text { Modes }\end{array}$ \\
\hline $20 \mathrm{~nm}$ & $2.9680(2.9680)$ & Cutoff & Cutoff & Cutoff & Cutoff & Cutoff & 1 \\
\hline $30 \mathrm{~nm}$ & $2.3451(2.3451)$ & Cutoff & Cutoff & Cutoff & Cutoff & Cutoff & 1 \\
\hline $40 \mathrm{~nm}$ & $2.0816(2.0816)$ & $1.4560(1.4560)$ & Cutoff & Cutoff & Cutoff & Cutoff & 2 \\
\hline $60 \mathrm{~nm}$ & $1.8655(1.8655)$ & $1.4969(1.4969)$ & Cutoff & Cutoff & Cutoff & Cutoff & 2 \\
\hline $200 \mathrm{~nm}$ & $1.6437(1.6438)$ & $1.5912(1.5913)$ & $1.4544(1.4544)$ & Cutoff & Cutoff & Cutoff & 3 \\
\hline $300 \mathrm{~nm}$ & $1.6154(1.6159)$ & $1.5895(1.5900)$ & $1.5130(1.5135)$ & Cutoff & Cutoff & Cutoff & 3 \\
\hline $500 \mathrm{~nm}$ & $1.5922(1.5949)$ & $1.5818(1.5848)$ & $1.5505(1.5535)$ & $1.4984(1.5013)$ & Cutoff & Cutoff & 4 \\
\hline $700 \mathrm{~nm}$ & $1.5819(1.5895)$ & $1.5763(1.5843)$ & $1.5594(1.5675)$ & $1.5311(1.5392)$ & $\begin{array}{c}1.4913 \\
\text { ( } 1.4993)\end{array}$ & Cutoff & 5 \\
\hline
\end{tabular}
obtained by the analytical method and commercial software COMSOL Multiphysics. Two methods almost make the same results and small error is caused by an approximation of the infinite thickness of the cladding in our theoretical model.

Table 1 Effective indexes of $\mathrm{TM}_{0}$ mode and $\mathrm{HE}_{\mathrm{m}}(\mathrm{m}=1,2,3,4,5)$ modes guided by silver nanowires (the work wavelength is $\lambda_{0}=633 \mathrm{~nm}$ and the relative permittivity of silver is $\varepsilon_{r 1}=-16.22+\mathrm{i} 0.52$ ) 


\section{Deepen the students' impression for various modes by combining COMSOL Multiphysics and the analytical model}

In order to help students to understand the results obtained by the analytical method, we use wave optical module of commercial software COMSOL Multiphysics for analyzing surface modes guided by silver nanowires with radius $a=700 \mathrm{~nm}$ at $633 \mathrm{~nm}, 785 \mathrm{~nm}, 1064 \mathrm{~nm}$ and 1550nm, respectively. Normalized $|\boldsymbol{E}|$ distribution of several surface modes are shown in Figure 2.For every surface mode, the electric field is strongest at the interface between the metal core and the dielectric cladding and exponentially decays along the direction perpendicular to the interface. The fundamental mode is $\mathrm{TM}_{0}$ mode, and the second mode is $\mathrm{HE}_{1}$ mode. Higher-order modes own more cycles along the angular direction. There are five different modes $\left(\mathrm{TM}_{0}, \mathrm{HE}_{1}, \mathrm{HE}_{2}, \mathrm{HE}_{3}\right.$, $\mathrm{HE}_{4}$ ) while the wavelength is 633nm. However, the metal nanowire with the same size only support two modes ( $\mathrm{TM}_{0}, \mathrm{HE}_{1}$ modes) while the wavelength is longer $(1550 \mathrm{~nm})$. This result can help student understanding the cut-off characteristics of guided wave structures. The number of modes supported by the same guided wave structure varies with the work wavelength.

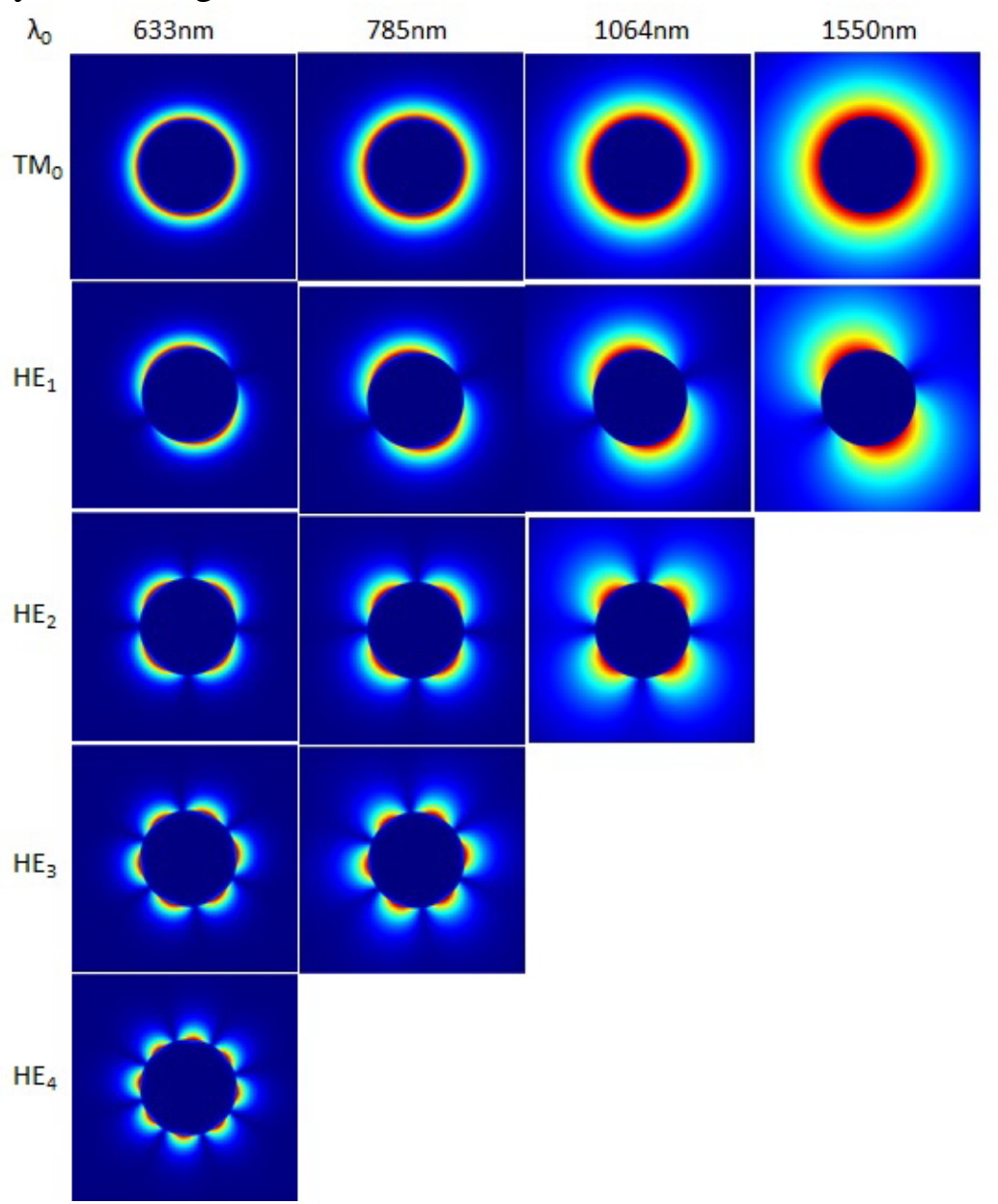

Fig 2. Normalized $|\boldsymbol{E}|$ distribution of several surface modes $\quad(a=700 \mathrm{~nm})$

\section{Conclusion}

All in all, introducing the latest research hotspots has greatly aroused students' enthusiasm to learn mathematical physics methods, stimulated the desire to use mathematical tools and basic knowledge for exploring the unknown world, and lay a solid foundation for learning basic courses. In the future, our research group will make efforts in the following aspects: (1) Make full use of existing software such as MATLAB, HFSS and COMSOL etc. and combine mathematical tools and current information technologies closely to enrich the classroom content; (2) Bring together the latest scientific issues and encountered practical problems of the communication field to the 
classroom. Systematical plan are needed for scientific research topics in our courses. (3) Enhance our own research level, and strive to introduce our latest research results to the classroom to enrich the teaching. (4) Encourage undergraduate to form their research group and take part in projects. In short, we will continue to adhere to the teaching reform, avoiding course content unchanged and avoiding the curriculum becoming a static data collection. We will gradually achieve the curriculum resource information "activation" and realize dynamic and renewable resources. In addition, we will keep communication with teachers of follow-up course for convergence of knowledge.

\section{Acknowledgement}

In this paper, the research was sponsored by National Natural Science Foundation of China (Project No. 61405083) and the Fundamental Research Funds for the Central Universities (lzujbky-2016-134, lzujbky-2016-135, lzujbky-2016-137).

\section{References}

[1] Zhang Jing, Zhang Yan, Tang Qiong, Xu Yuan-ying, Yang Yan-fang, "Mathematical Physics Method" course reform exploration. University education, 2014(16): p. 163-165.

[2] Liu Yongluo, exploration and thinking of research-based teaching of theoretical physics course. Journal of Higher Education Research, 2013. 36(S1): p. 80-82.

[3] Wang X, Liu Xiongwei, "Exploration and Practice of Research-oriented Teaching of Differential Equations", Journal of Higher Education Research, 2013(2): p. 27-30.

[4] Mei Zhonglei, Xu Fuyong, Cao Binzhao, and Li Yuee, Tracking the Frontier of Science and Technology, Servicing Course Teaching --- The Practice and Approach of Research - based Teaching in the Course of "Electromagnetic Field Theory". Higher Education of Sciences,2008, 82 (6) : 80-83.

[5] Rashid Zia, Jon A. Schuller, Anu Chandran, Mark L. Brongersma, plasmonics: the next chip-scale technology. Materials Today, 2006. 9(7-8): p. 20-27.

[6] Park J, Kim KY, Lee IM, Na H, Lee SY, Lee B., Trapping light in plasmonic waveguides. Optics Express, 2010. 18(2): p. 598-623. 